\title{
Evaluation of Adaptation to Adverse Working Conditions in Different Regions of Russia
}

\author{
IV Yatsyna ${ }^{1}$, ${ }^{*}$ LM Saarkoppel ${ }^{2}$, EN Kryuchkova ${ }^{3}$ and AV Istomin ${ }^{4}$ \\ ${ }^{1}$ Director of the Institute of General and Occupational Pathology F.F. Erisman Rospotrebnadzor, Russia \\ ${ }^{2}$ Academic degree, title - Doctor of Medical Sciences, Professor Position - leading researcher of the Institute of General and Professional \\ Pathology, Russia \\ ${ }^{3}$ Department of Laboratory Diagnostics, Senior Researcher, Russia \\ ${ }^{4}$ Academic degree, title - Doctor of Medical Sciences, Professor Position - Head. healthy and safe nutrition department, Russia \\ *Corresponding author: IV Yatsyna, Director of the Institute of General and Occupational Pathology FF Erisman Rospotrebnad- \\ zor, Russia
}

\section{ARTICLE INFO}

Received: 幽 July 31, 2019

Published: 幽 August 12, 2019

Citation: IV Yatsyna, LM Saarkoppel, EN Kryuchkova, AV Istomin. Evaluation of Adaptation to Adverse Working Conditions in Different Regions of Russia. Biomed J Sci \& Tech Res 20(3)-2019. BJSTR. MS.ID.003459.

Keywords: Adolescents; Miners; Functional Potential; Systems of Homeostasis; Coefficient of Correlation Adaptation; Specialized Product of Dietary Preventive Nutrition; Medical and Biological Efficacy

\section{ABSTRACT}

The problem of preserving the health of industrial workers is especially significant in the northern regions of Russia, where mining remains one of the leading industries. Climategeographical conditions of the Arctic zone contribute to a higher voltage adaptation system of the body and lead to an increase in overall morbidity, which is higher in the northern regions than in the whole country. The purpose of the study is to assess the functional potential of children and adults in the northern regions of Russia and the Central zone and ways to increase it.

Materials and Methods: Comparative studies were carried out at the mining enterprises of the Norilsk industrial region and the Belgorod region. Were also examined teenagers (16-17 years old), living in areas of the Arctic zone and central Russia. To determine the changes occurring in the body of workers and adolescents under the influence of negative environmental factors, indicators of protein, lipid, mineral, vitamin exchanges and antioxidant status were used.

Results: A methodical approach was developed to assess the health of workers in adverse industrial conditions based on determining the degree of correlation interrelations between the indices of homeostasis of the organism. The proposed integral indicator - the coefficient of correlation adaptation (CAC) - allows you to determine the level of adaptation of the surveyed populations to the impact adverse factors, establish a timeline for the risk of developing health problems, timely determine and differentiate the directions of preventive measures. The proposed method is used to assess the functional state of the body of adolescents in contrast regions. In order to increase the body's resistance to adverse effects of harmful factors, a specialized dietary product has been applied. preventive nutrition "Leovit" diet (4.5) under harmful working conditions. The efficiency of the product use was confirmed by the normalization of homeostasis parameters in more than $80 \%$ of the examined and an increase in the CCA from the level of "reduced functional potential" $(0.19)$ to the optimal level (0.33).

\section{Introduction}

Preserving the health of the working population as the most important productive force of society is one of the most important state tasks. Among the many factors that shape the health of the working-age population, the quality of the environment plays the most important role: working conditions, life conditions, food, lifestyle, and the state of the environment. Addressing the 
issues of the formation and preservation of health in the extreme climatic and geographical conditions of the Arctic zone, being the richest raw material base, it is especially important against the background of the socio-economic changes taking place in the country, which brought to the fore the significance of the regions of the Arctic zone in the Russian economy [1-4]. The climatic conditions of the polar regions contribute to the development in the human organism of rearrangements of many functional systems, forming a qualitatively new state of the organism - an adaptation that is achieved at the price of a certain biosocial charge. The state of adaptation can be described as the process of maintaining the functional state of the homeostatic systems of the body, ensuring its preservation, development, efficiency, maximum longevity in adequate and inadequate environmental conditions [5-8]. Working conditions in mining operations (high levels of noise, vibration, dust, uncomfortable microclimate, severe physical and psychophysiological loads) lead not only to the formation of occupational pathology, but also to a decrease in the overall resistance of the organism $[9,10]$.

Nutrition plays an important role in human adaptation to the conditions of the north, since body energy costs increase. In extreme conditions of the Arctic zone, all types of metabolism are involved in the long process of adaptation - protein, carbohydrate, fat, microelement, vitamin. Food and its individual components is an effective means of increasing the body's resistance to the adverse effects of the external environment and thus, perform an adaptive and regulatory function, which is especially important for the regions of the Arctic zone, where a person is exposed to a whole range of negative environmental factors. By scientific justification of nutrition in specific living and working conditions, it is possible to significantly affect the functional state of the body and prevent disease $[5,6,8,11-17]$. At the same time, at the present stage, the state of health of the younger generation is of great importance. It has to start working in mines, metallurgical, chemical and other enterprises, where professional selection is carried out for medical reasons. On the one hand, the children's organism, due to the limitedness of its adaptive capabilities, is more sensitive to the action of adverse environmental factors. On the other hand, the adaptive reactions of a child's body are formed, as a rule, in the usual climatic zone and habitat $[3,7,10]$. In recent years, interest in methods of integral quantitative assessment of indicators of the functional state of the body has increased significantly. One of the promising methods for assessing the adaptation reserves of the organism in a population is the method of correlation adaptometry, which allows us to characterize various aspects of relationships in a variety of variables. The main idea of the method is to demonstrate the position that informational relationships both within individual functional systems and in intersystem connections in the whole organism are very sensitive to external influences $[9,12]$. Long-term studies based on a comparative analysis of populations and groups in various environmental and socio-hygienic conditions indicate a highly informative correlation between physiological parameters to assess the degree to which the population is adapted to extreme or simply changed conditions. Thus, the purpose of this study was to make a comparative assessment of the functional potential of children and adults in the Arctic zone of Russia and ways to increase it in order to preserve the labor potential of the population.

\section{Materials and Methods}

The research was carried out at the leading enterprises of the mining industry of the Norilsk industrial region and the Belgorod region. In the period 2009-2015. In-depth clinical and laboratory examination of 876 miners of the Far North and Middle Zone (642 workers of OJSC MMC Norilsk Nickel; 234 workers of OJSC KMAruda) with work experience from 0.5 to 25 years. To determine the initial level of the functional state of the body of the working population, 140 older children (16-17 years old) of school age of the respective contrast climatic and geographical regions were examined. To verify the changes occurring in the workers' body under the influence of negative factors of the working environment, indicators of peripheral blood, protein $(\mathrm{OB}$, protein fractions, glycosaminoglycans, fibrinogen, D-dimers), lipid (OX, TG, OH-HDL, OH-LDL ), mineral (Ca, P, Fe, Mg, Zn, Cu), vitamin (ascorbic acid, retinol, $\alpha$-tocopherol, etc.) exchanges, antioxidant status (MDA, catalase, SOD, neutrophil myeloperoxidase, ceruloplasmin) and hormonal status (thyrotropic hormone, triiodothyronine, thyroxin, cortisol, adrenocorticotropic hormone, testosterone, luteinizing hormone, aldosterone); cellular (subpopulations of lymphocytes $\mathrm{CD} 3+, \mathrm{CD} 4+, \mathrm{CD} 8+, \mathrm{CD} 16+, \mathrm{CD} 20+$ ) and humoral immunity were assessed (immunoglobulins IgA, IgM, IgG, IgE, CIC, etc.), cytokine levels (interleukins IL-2, IL-4, IL-8 , IL-1 $\beta$, TNF- $\alpha$ ) [4]. There are 70 parameters defined. Laboratory indicators of leading homeostasis systems are selected based on the analysis of literature data on the effect of the main production factors on the human body. In the course of experimental studies, an evaluation was carried out of the medical and biological effects of specialized products of LEOVIT Nutrio LLC (Moscow) -dietary preventive jelly. A worker in the conditions of production was given a BOB (diet 4.5) under harmful working conditions in the amount of 2 tbsp. spoons for 1 cup of hot water per day. The scheme included the determination of the above indicators of peripheral blood, immune and antioxidant status, lipid, mineral exchanges before the start of preventive measures, after 4, 12 weeks and 6 months of consumption of a functional product. Mathematical processing was carried out using Microsoft Excel 2003, Statistica 6.0 and StatPlus Professional 2008 software packages. The correlation and regression analysis methods were used to assess the relationship between the studied indicators.

\section{Results and Discussion}

For a quantitative assessment of the functional reserves of the body of workers, we developed a method using the integral correlation adaptation coefficient (CAC), determined by the degree of conjugacy of diagnostic indicators of the main systems 
of homeostasis using the pair correlation method [16]. The basis of the proposed approach is the definition of contingency indicators leading systems of homeostasis, responsible for adapting the body to changes in the external environment using a set of diagnostic methods, primarily biochemical, as well as immunological and cytochemical, with highly informative. The number of reliable correlations in the total number of correlation coefficients considered was determined and their degree of severity was estimated by the weight of the correlation graph $\mathrm{G}$, calculated as the sum of reliable correlation coefficients without considering their directivity (Figure 1).

$$
\begin{gathered}
G=\sum \text { I rijI } \\
r i j \geq \alpha
\end{gathered}
$$

where rij - correlation coefficients between i and j indicators, $\alpha$ - is determined by the level of confidence $(0.3 \leq \mathrm{rij} \leq-0.3)$. Based on the substantial variability of the magnitude of the correlation graph depending on the number of studied parameters, a correlation adaptation coefficient (CCA) was proposed, calculated on the basis of research data and allowing quantitative characterization of functional deviations in groups of subjects.
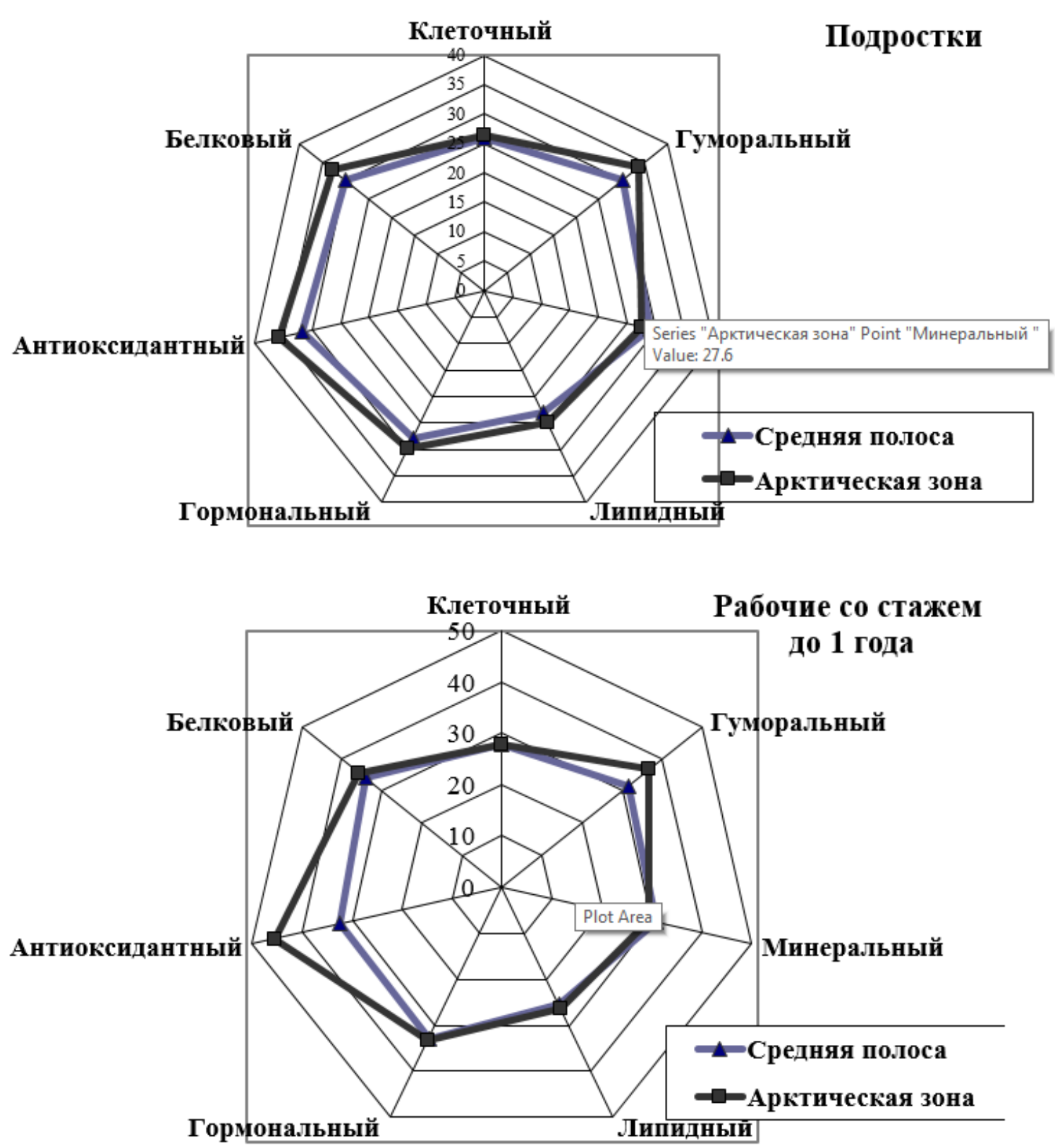

Figure 1: Assessment of the strength of the relationship between the indicators of various systems of homeostasis in adolescents of the middle zone and the Arctic zone of the Russian Federation. 


\section{$K K A=G a v / N$}

where $\mathrm{N}$ is the number of studied parameters. Four levels of functional capacity were established to identify groups and "probation zones" of health risk: "optimal" (Ck $\geq 0.3)$; "Satisfactory" $(0.2 \leq \mathrm{CCA}<0.3)$; "Decrease in functional potential" $(0,1 \leq \mathrm{KKA}<0,2)$; "Depletion of functional potential" (CCA $<0.1$ ). The need to study the functional state of the body of adolescents corresponding to contrasting climatic and geographical regions is argued that the functional state of the body of an adult and its reserves are formed on the basis of the functional potential of the teenage body. In addition, the children's population is the most sensitive contingent, quickly responding to any environmental changes due to the incompleteness of the processes of growth and development.

When comparing clinical and laboratory parameters in adolescents of the north and the middle band, it was revealed that changes in the T-cell immunity were characterized by activation of T-lymphocytes and T-helper cells in both groups. This suggests the presence of antigenic stimulation of the immune system. At the same time, a decrease in T- suppressors. It should be noted that B-lymphopenia was more common in northerners than middle school students $(15 \%$ and $7 \%$, respectively, $\mathrm{p}<0.001)$. Studies of the humoral immunity of adolescents revealed elevated levels of IgM in the blood of northerners. The results obtained testified to the formation of defects in the immune defense, a violation of the immune regulation leading to the state of adaptive stress. Reducing the reserve capacity of immune regulation determines the inhibition of age development of the immune system in children with the subsequent formation of immunodeficiency states in the adult population, which was confirmed in our study in the study of immunological parameters in the examined workers. Along with this, schoolchildren of the Arctic zone found a decrease in mineral metabolism (Ca, Mg, $\mathrm{Zn}, \mathrm{Cu}$ ) in $25-40 \%$ of cases, vitamins (A, C, E) in $20-60 \%$. An increase in POL products in $44 \%$ of adolescents due to an imbalance in the antioxidant protection system was noted: increase in catalase in $56 \%$ of cases, CP - in 30\%, decrease in MP - in $45 \%$. In adolescents in the middle zone, the deviations of the studied parameters were 1.5-2 times less frequent.

The use of the method of correlation adaptometry made it possible to state the regional characteristics of the adaptation process in adolescents. An analysis of the conjugacy of immunological and biochemical parameters, reflecting the studied systems of a nonspecific response of the organism to the effects of adverse environmental factors, showed moderate correlations (from $r \pm 0.35$ to 0.69 ) between them. Despite the identified changes in the clinical and laboratory parameters of the studied systems in adolescents from different regions, their functional potential was quite high and corresponded to the optimal level. The average weight of the correlation graph $G$ and the value of the correlation coefficient Kka's adaptations among schoolchildren of the north were slightly higher (30.2 and 0.53 , respectively) than among middle-aged adolescents (28.3 and 0.5), which reflects the mobilization of the functional reserves of the body of northerners to the influence of extreme factors. Similar changes were found in miners in the studied regions with experience of up to 1 year (Figure $1)$. When comparing the dynamics of changes in the coefficient of correlation adaptation of the CAC among the workers of the studied production, depending on the length of service, a number of features were identified. At the beginning of work (experience up to 1 year; age $25.0 \pm 0.3$ years), the level of functional reserves in all the examined groups corresponded to the optimum (Kka $=0.44$ 0.49 ) and was close to the level of adolescents (16-17 years) Kka = $0.5-0.53$ of the respective regions.

In the same way as in the northerly adolescents, the value of the CAC was higher among the workers of the North (0.49). With an increase in the length of service, both in miners of the Norilsk region and in the middle zone, the CCA gradually decreased from the optimal to a satisfactory level of functional potential (with length of service up to 5 years - 0.41 and 0.36 ; with length of service up to 10 years $-0,32$ and 0.29 respectively) (Figure 2). With an experience of 11-15 years, miners of the MMC Norilsk Nickel, the functional reserves of the body are sharply reduced (CCA $=0.026)$, and the miners of OJSC KMA-ore - with an experience of 16-20 years (CCA $=0.029)$, which corresponds to the depletion of functional potential and, therefore, disruption of the normal adaptation process. With the help of correlation adaptometry, it is possible not only to identify the need for preventive measures even before the onset of symptoms of the disease among members of any group of people, but also to evaluate their effect on reducing adaptive stress. One of the promising ways to increase the body's resistance to the adverse effects of harmful factors is the introduction of specialized products that help strengthen the adaptive reserves of workers. Against the background of the use of a specialized product of preventive nutrition for 4 weeks, the frequency of detection of polyhypovitaminosis states decreased 1.3 times. 


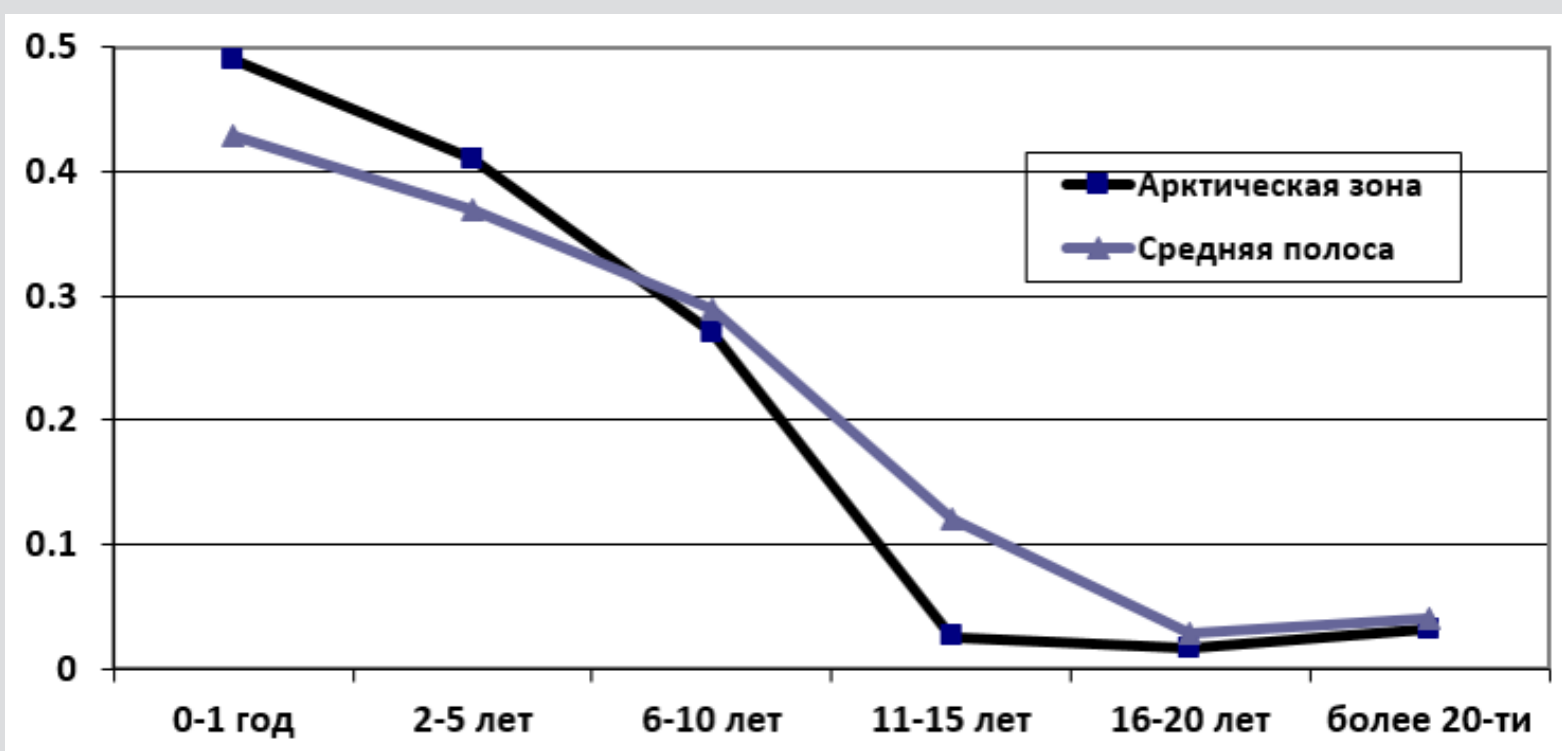

Figure 2: The dynamics of the coefficient of correlation adaptation depending on the length of service of miners.

Certain shifts in the improvement of the antioxidant defense system (POL-AOD) were noted. However, despite this, in the surveyed miners, the coefficient of correlation adaptation of the CAC increased slightly from 0.16 to 0.19 within one level (reduced functional potential), which characterizes the lack of effectiveness of a 4-week course of use of a specialized therapeutic and prophylactic product for optimal correction of functional potential. A 12-week course of correctional nutrition promoted immunomodulatory, anti-inflammatory effects, optimization of indicators (POL-AOD) and an increase in the functional reserves of the body of miners. The coefficient of the CAC has increased from 0.2 to 0.26 within the same level, corresponding to a satisfactory functional potential, which characterizes the effective, but insufficient for the duration of the use of therapeutic and prophylactic product [17-19]. The effectiveness of a 6-month course of application of a therapeutic and prophylactic product under production conditions is confirmed by the correction of metabolic processes by supplementing by $2540 \%$ of mineral and vitamin deficiency, a decrease in the accumulation of POL products by $20 \%$ with activation of the enzyme component of antioxidant protection by 1.2-1.3 times, optimization of cellular and humoral immunity indicators(normalization of CD4 + / CD8 + immunoreactivity coefficient; reduction of IgM and G levels to 1.4 times) in the studied contingent of workers. In the process of consumption of the specialized product Leovit, the coefficient of correlation adaptation of the CCA increased, characterizing the increase in the functional reserves of the body of the miners. The functional potential of the source was assessed as reduced (CCA = 0.19). After 3 months, nutritional support for the CCA increased to 0.27 (satisfactory functional capacity). As a result of 6 months of preventive measures, the level of functional potential increased to the optimum $(\mathrm{CCA}=0.33)$.

\section{Conclusion}

Thus, the developed methodological approach to the study of the adaptation of working contingents to the effects of adverse environmental factors with the definition of an integral indicator - the correlation coefficient of adaptation (CCA) allowed a comparative assessment of the adaptation levels of workers in contrasting climatic and geographical regions, timely determine and differentiate the direction of preventive measures. The dynamics of the CCA integral index in the surveyed groups of workers against the background of nutritional support of various duration testified to the reliable effectiveness of the 6-month rehabilitation course and the use of the developed methodological approach to the assessment of functional potential. The timely use of a complex of medical and preventive measures, possessing high efficiency, optimizes the functional reserves of the body under the influence of adverse environmental factors and contributes to the preservation of the labor potential of the population of various industrial regions.

\section{References}

1. Baevsky RM, Berseneva AP (2009) Using the principles of prenosological diagnostics to assess the functional state of the body under stress effects. Human Physiology: Journal of the Russian Academy of Sciences 35(1): 41-51.

2. Boyko ER (2007) Physiological and biochemical basis of human activity in the North. Ekaterinburg, 190s.

3. Vinyarskaya IV (2007) Indicators of the quality of life of healthy adolescents living in different regions of Russia. Public Health and Health Care 3: 37-40.

4. Degteva GN, Zubov LA (2004) Actual issues of social, physiological and metabolic adaptation of the human body to the conditions of the North. Human Ecology 4: 57-59.

5. Istomin AV, Saarkoppel LM, Yatsyna IV (2015) Hygienic problems of correction of the power factor for workers in hazardous conditions. Moscow: Dashkov and Co pp. 186. 
6. Kryuchkova EN (2008) Efficiency of alimentary correction of maladaptation shifts among miners in the Middle zone. Healthcare of the Russian Federation p. 44-45.

7. Kuchma VR (2009) Physical development, health and lifestyle of children of the Circumpolar. M .: Medicine pp. 194.

8. Pilat TL, Istomin AV, Baturin AK(2006) Food workers in harmful and particularly harmful working conditions. History and state of the art M pp. 240.

9. Razhevaykin VN, Shpitkov MI (2006) Application of the method of correlation adaptometry in biomedical problems. p. 3-13.

10. Rakhmanin Yu A (2010) An integrated approach to the hygienic assessment of the quality of life of students. Hygiene and Sanitation pp. 67-69.

11. Saarkoppel LM, Serebryakov PV, Fedina IN (2005) Ecological and hygienic health problems of miners in the Norilsk region. Ed. Academician of RAMS, prof. Potapova AI M “Science pp. 224.

12. Sokolov AV (2007) The role and place of the integral assessment of the functional reserves of the body in restorative medicine. Spa business pp: $5-10$.

ISSN: 2574-1241

DOI: 10.26717/BJSTR.2019.20.003459

IV Yatsyna. Biomed J Sci \& Tech Res

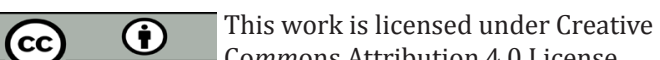

Submission Link: https://biomedres.us/submit-manuscript.php
13. Syurin S, Shilov VV (2016) Features of health disorders of miners of the northern copper-nickel mines. Hygiene and Sanitation 95(5): 455-459.

14. Tutelyan VA (2009) State policy of healthy nutrition of the population: tasks and ways of implementation at the regional level M GEOTAR-Media pp: 288.

15. Ushakov IB, Sorokin OG (2004) Human Adaptation Potential. Bulletin of the Russian Academy of Medical Sciences 3: 8-13.

16. Yudina TV, Saarkoppel LM, Kryuchkova EN (2016) An integrated approach to assessing the health of workers in hazardous industries. Healthcare of the Russian Federation 60(2): 101-104.

17. Burström L, Aminoff A, Björ B, Mänttäri S, Nilsson T, et al. (2017) Arculoskeletal disease in the arctic. International Journal of Occupational Medicine and Environmental Health 30(4): 553-564.

18. MacGillivray DM, Kollmann TR (2014) The role of environmental factors in modulated immune responses in early life. Front Immunol 5: 434-440.

19. Young TK, Makinen TM (2010) The Health of Arctic Populations: Does Cold Matter. Am J Hum Biol 22(1): 129-133.

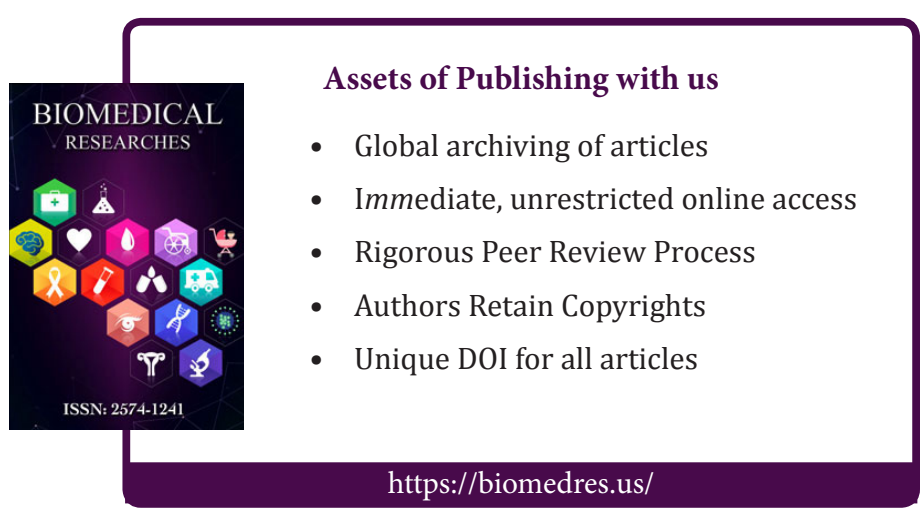

